\title{
Effect of STAT3 on Lysophosphatidic Acid-Induced Oral Cancer Cell Invasion
}

\author{
Zi Hae Song, Kyung Hwa Cho, Jin Young Kim, and Hoi Young Lee ${ }^{\dagger}$ \\ Department of Pharmacology, College of Medicine, Konyang University, Daejeon 35365, Korea
}

\begin{abstract}
Background: Oral cancer has a high incidence worldwide and has been closely associated with smoking, alcohol, and infection by the human papillomavirus. Metastasis is highly important for oral cancer survival. Lysophosphatidic acid (LPA) is a bioactive lipid mediator that promotes various cellular processes, including cell survival, proliferation, metastasis, and invasion. Signal transducer and activator of transcription (STATs) are transcription factors that mediate gene expression. Among the seven types of STATs in mammals, STAT3 is involved in invasion and metastasis of numerous tumors. However, little is known about the role of STAT3 in oral tumor invasion. In the present study, we hypothesized that STAT3 mediates LPA-induced oral cancer invasion.

Methods: Immunoblotting was performed to analyze LPA-induced STAT3 activation. 3-(4,5-Dimethyl-2-thiazolyl)-2,5-diphenyl-2H-tetrazolium bromide (MTT) assay was performed to assess the survival rates of YD-10B cells. STAT3 levels in LPA-treated oral tumor cells were evaluated by performing in vitro invasion assay.

Results: To the best of our knowledge, this is the first study to demonstrate that LPA enhances STAT3 phosphorylation in oral cancer. In addition, treatment with WP1066, a selective inhibitor of STAT3, at a concentration that does not cause severe reduction in cell viability, significantly attenuated LPA-induced YD-10B cancer cell invasion.

Conclusion: The results suggested that LPA induces oral tumor cells with greater invasive potential via STAT3 activation. Our findings provided important insights into the mechanisms underlying mouth neoplasms.
\end{abstract}

Key Words: Epithelial-mesenchymal transition, Lysophospholipids, Mouth neoplasms, STAT3 transcription factor

\section{Introduction}

Head and neck cancers are common diseases worldwide that affect the squamous cells in the oral cavity, pharynx, and larynx, out of which oral cancer accounts for the majority of cases ${ }^{1,2)}$. In 2018, 354,864 people developed oral cancer, and 177,384 of them died ${ }^{3)}$. Oral cancer occurs in the tongue, lips, buccal mucosa, gingiva, and palatal mucosa. Tongue cancer is the most common cancer in Korea and is more prevalent among men than women over the age of $60^{4)}$. The causes of oral cancer include smoking, alcohol drinking, diet, mouth wash, Paraguay tea, and human papillomavirus infection ${ }^{5,6}$. The overall survival rate and disease-free survival rates for oral cancer have been reported to be $56 \%$ and $58 \%$, respectively ${ }^{7)}$. Metastasis severely impacts the survival of oral cancer patients, and patients with extracellular metastasis have shorter lifespans compared to patients with intranodal metastasis ${ }^{8}$.

Lysophosphatidic acid (1- or 2-acyl-lysophosphatidic acid, LPA) is a lipid mediator that is produced in the vast majority of mammals and is known to be involved in various intracellular activities ${ }^{9}$. The binding between LPA and $\mathrm{G}$ protein-coupled receptor in cancer cells is known to contribute to the growth, migration, and metastasis of tumors $^{10)}$. Furthermore, LPA has been demonstrated to facilitate osteoclast formation by inducing the release of interleukin (IL) 6 and IL-8 in oral squamous cell carcinoma ${ }^{11)}$. 
Recently, our group reported that LPA plays an important role in promoting the metastasis of oral cancer cells by upregulating the expression levels of TWIST1 and Slug ${ }^{12,13)}$. However, the exact mechanism by which LPA induces oral cancer metastasis remains to be elucidated. Signal transducer and activator of transcription (STAT) protein acts as a transcription factor ${ }^{14)}$. Mammals have seven types of STAT proteins, and STAT3 activation is closely related to tumor growth and metastasis ${ }^{15)}$. STAT3 has been reported to be involved in the progression of tongue squamous cell carcinoma ${ }^{16)}$, and inhibition of STAT3 was reported to inhibit invasion of oral cancer cells $^{17)}$. Previously, we showed that LPA induces STAT3 phosphorylation and facilitates cellular migration in ovarian cancer cells ${ }^{18)}$. However, the LPA-involved STAT3 signaling pathway in oral cancer remains to be investigated. Therefore, the present study aimed to examine the association between LPA and STAT3 in the invasion of oral cancer cells.

\section{Materials and Methods}

\section{Reagents}

LPA was purchased from Avanti Polar Lipids (Alabaster, AL, USA). WP1066 (Calbiochem, La Jolla, CA, USA) and dimethyl sulfoxide (DMSO) were purchased from SigmaAldrich (St. Louis, MO, USA). The primary antibodies p-STAT3 (1:1,000 dilution) and STAT3 (1:1,000 dilution) were obtained from Cell Signaling Technology (Danvers, MA, USA). Glyceraldehyde 3-phosphate dehydrogenase (GAPDH, 1:3,000 dilution) antibody was purchased from Santa Cruz Biotechnology Inc. (Santa Cruz, CA, USA). All reagents used were of the purest grade.

\section{Cell culture}

Oral squamous carcinoma YD-10B cells were kindly provided by Dr. J.I. Yook (Yonsei University College of Dentistry, Korea). YD-10B cells were cultured in Hyclone RPMI 1640 medium containing 10\% fetal bovine serum (FBS; Hyclone, Logan, UT, USA) and $1 \%$ penicillin/ streptomycin (Lonza, Allendale, NJ, USA) with $5 \% \mathrm{CO}_{2}$ atmosphere at $37^{\circ} \mathrm{C}$. After reaching $80 \%$ confluence on a T75 plate, the cells were washed twice with phosphate buffered saline (PBS) solution and treated with trypsinethylenediaminetetraacetic acid (EDTA; Lonza) for subcultures.

\section{Cell viability assay}

YD-10B cells were subcultured in a 96-well plate at $4 \times 10^{4}$ cells per well, and the cells were treated with serum-free medium containing WP1066 for 12 hours. Cells were washed with PBS (ATTO Corp., Tokyo, Japan) and treated with $5 \mathrm{mg} / \mathrm{ml} \mathrm{3-(4,5-Dimethyl-2-thiazolyl)}$ -2,5-diphenyl-2H-tetrazolium bromide (MTT; SigmaAldrich) for 2 hours. After washing with PBS, $100 \mu$ of DMSO was added to each well, and the absorbance was measured at $540 \mathrm{~nm}$ using an ELISA plate reader (BioTek Instruments Inc., Winooski, VT, USA).

\section{Immunoblotting}

After culturing and stabilizing YD-10B cells in a $60 \mathrm{~mm}$ dish, cells were treated with serum-free medium containing $2.5 \mu \mathrm{M}$ WP106. After 12 hours of incubation, the experimental group was treated with LPA, washed with cold PBS, and then added with radioimmunoprecipitation (RIPA; Roche, Basel, Switzerland) buffer for protein extraction. The cells were scraped with a scraper into a $1.5 \mathrm{ml}$ tube and centrifuged at $4^{\circ} \mathrm{C} 13,000 \mathrm{rpm}$ for 15 minutes. The supernatant was transferred to a new $1.5 \mathrm{ml}$ tube, and the protein concentration was measured using bicinchoninic acid protein assay reagent (Thermo Fisher Scientific Inc., Waltham, MA, USA). The proteins were separated according to size by SDS polyacrylamide gel electrophoresis (PAGE), transferred to polyvinylidene difluoride membranes (PVDF; Merck Millipore, Burlington, MA, USA), blocked with EzBlockChemi (ATTO Corp.), and then diluted at $1 / 10$ for 30 minutes. To allow binding of the primary antibodies, the membrane was washed thrice with $1 \times$ TBST $(0.1 \%$ Tween 20 in Tris-buffered saline [TBS]) and left to stand at $4^{\circ} \mathrm{C}$ overnight. The next day, the membrane was washed with $1 \times$ TBST and incubated with a secondary antibody at room temperature for 2 hours. After washing with $1 \times$ TBST for 30 minutes, enhanced chemiluminescence reagent (Pierce, Rockford, IL, USA) was added to verify the presence of the proteins using Image Quant 300 (GE Healthcare, Buckinghamshire, 
UK). Immunoblotting was performed using anti-rabbit and anti-mouse secondary antibodies from Thermo Fisher Scientific Inc. (Rockford, IL, USA).

\section{In vitro invasion assay}

In vitro invasion assay was performed using a membrane coated with Matrigel (BD Biosciences, San Jose, CA, USA) and a Boyden chamber (Neuro Pro Inc., Gaithersburg, MD, USA). In the lower chamber, $27 \mu$ of serum-free medium containing $0.1 \%$ bovine serum albumin (SigmaAldrich) or $5 \mu \mathrm{M}$ LPA was added. In the upper chamber YD-10B $\left(5 \times 10^{5}\right.$ each $)$ cells treated with DMSO for the control group or treated with WP1066 for the experimental group were added and subsequently incubated at $37^{\circ} \mathrm{C}$ for 14 hours. On the next day, the cells dyed with Diff-Quik reagent (Dade Behring Inc., Newark, DE, USA) were counted on a microscope at $200 \times$. The results are presented in a graph.

\section{Statistical analysis}

Results of cell viability and in vitro invasion assay were analyzed by t-test. p-value $<0.05$ was considered statistically significant. All results were analyzed using the IBM SPSS Statistics ver. 22.0 (IBM Corp., Armonk, NY, USA) software. Data were presented as mean \pm standard deviation.

\section{Results}

\section{Lysophosphatidic acid induces STAT3 phosphorylation}

STAT3 phosphorylation is known to be intimately associated with the progression of oral cancer ${ }^{19)}$. Therefore, we first examined whether LPA phosphorylates the tyrosine

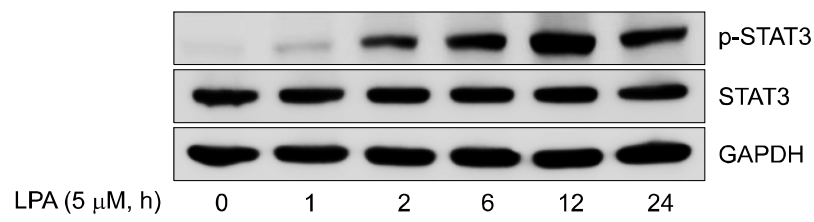

Fig. 1. STAT3 activation induced by LPA. Immunoblotting analysis was performed with YD-10B oral cancer cells. YD-10B cells were pre-treated with LPA in a time-dependent manner after serum starvation for 12 hours. The membranes were probed with specific primary antibodies against anti-pSTAT3, anti-STAT3, and GAPDH. GAPDH was used as a loading control. LPA: Iysophosphatidic acid, GAPDH: glyceraldehyde 3-phosphate dehydrogenase. residue of STAT3. Oral cancer cells YD-10B were treated with $5 \mu \mathrm{M}$ LPA at each time point to extract proteins, and STAT3 phosphorylation levels were examined via immunoblotting. Results showed that the protein levels of p-STAT3, the active form of STAT3, were elevated after LPA treatment, and STAT3 activity peaked at 12 hours (Fig. 1). These results confirmed that LPA induces STAT3 activation in oral cancer cells.

\section{STAT3 inhibitor at the concentration of $2.5 \mu \mathrm{M}$ does} not inhibit the survival of oral cancer cells

WP1066 is a selective inhibitor of STAT3 and has been known to inhibit oral cancer cell growth ${ }^{20)}$. Therefore, we attempted to identify the concentration at which WP1066 suppresses STAT3 while maintaining the viability of YD-10B oral cancer cells. Cells were treated with varying concentrations of WP1066, and cell viability was evaluated by the MTT assay. Results revealed that YD-10B survival rates at each specified concentration were $97.74 \%, 90.55 \%$, $71.77 \%$, and $51.3 \%$, and $2.5 \mu \mathrm{M}$ was the concentration at which WP1066 suppresses the migration of cells while allowing more than $85 \%$ survival rate (Fig. 2). Hence, cells were treated with $2.5 \mu \mathrm{M}$ WP1066 in subsequent experiments.

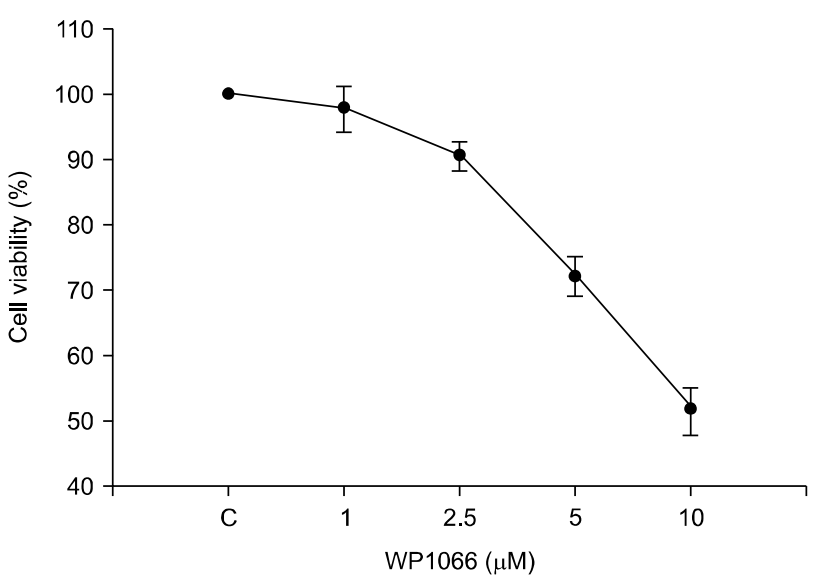

Fig. 2. Effects of WP1066 on cell viability. 3-(4,5-Dimethyl-2-thiazolyl)-2,5-diphenyl-2H-tetrazolium bromide (MTT) assay was performed to analyze cell viability. WP1066 affects cell viability in a dose-dependent manner. Oral cancer YD-10B cells showed $85 \%$ survival after treatment with $2.5 \mu \mathrm{M}$ WP1066. 


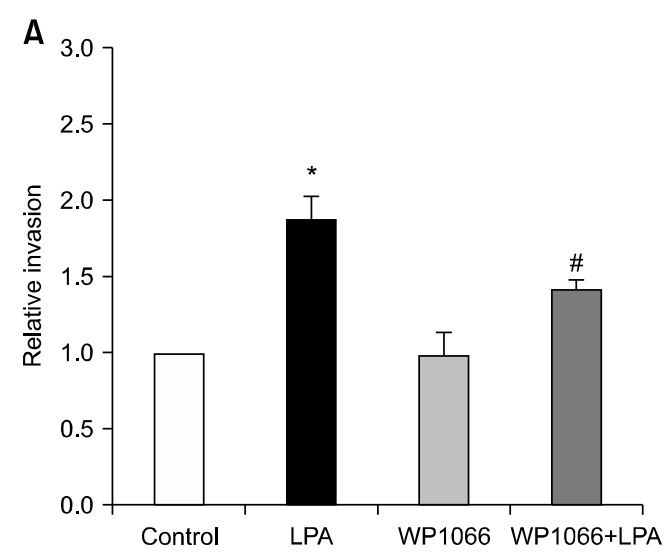

B

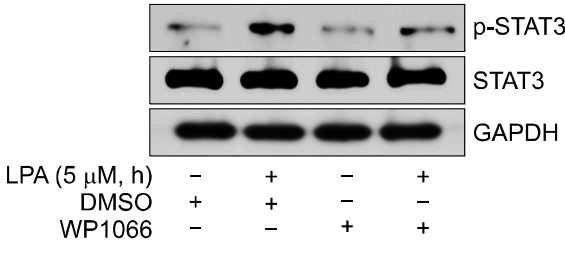

\section{STAT3 is crucial for lysophosphatidic acid-induced} oral cancer cell invasion

To examine the impact of selective inhibition of STAT3 on LPA-induced oral cancer cell invasion, we performed an in vitro invasion assay using a Boyden chamber. Results showed that cell migration elevated by LPA treatment was significantly inhibited when cells were treated with WP1066, a STAT3 inhibitor (Fig. 3). Therefore, LPA-induced oral cancer cell invasion is closely related to STAT3.

\section{Discussion}

LPA is produced by autotoxin and is known to promote various cellular activities, including carcinogenesis and cancer progression, by binding to an LPA receptor ${ }^{10)}$. Studies have demonstrated that LPA facilitates osteoclast formation via ILs in oral squamous cell carcinoma ${ }^{11)}$. In addition, one study reported that LPA facilitates cell invasion by binding to LPA receptor 3 in oral cancer ${ }^{21)}$. However, the exact mechanism by which the lipid mediator LPA promotes oral cancer cell invasion remains to be investigated. Therefore, this study examined LPA-induced STAT3 activity and is the first to confirm that STAT3 is crucial for LPA-induced oral cancer cell invasion. Immunoblotting results confirmed that LPA induces STAT3 phosphorylation in YD-10B oral squamous cell cancer cells, which indicated that LPA increases STAT3 activation. Given that STAT3 plays an important role in the invasion and metastasis of various carcinomas, including oral cancer ${ }^{15)}$, the finding that LPA increases STAT3 activity suggested that STAT3 is involved in the
Fig. 3. WP1066 inhibits LPA-induced oral cancer cell invasion. Modified Boyden-chamber was utilized to analyze the invasion of $Y B$ $10 \mathrm{~B}$ cells against LPA after incubation with or without WP1066 for 1 hour. $0.1 \%$ BSA and DMSO were used as solvent to melt LPA and WP1066, respectively. (A) In vitro invasion assay. Error bars: \pm standard deviation. $* \mathrm{p}<0.05$ vs. control, ${ }^{\#} \mathrm{p}<$ 0.05 vs. LPA treatment. (B) Immunoblotting. LPA: Iysophosphatidic acid, GAPDH: glyceraldehyde 3-phosphate dehydrogenase, DMSO: dimethyl sulfoxide, BSA: bovine serum albumin. migration of oral cancer cells induced by LPA. In addition, MTT assay confirmed that the optimal WP1066 (STAT3 inhibitor) concentration at which oral cancer cell migration is inhibited without affecting cell viability is $2.5 \mu \mathrm{M}$. These results suggest that WP1066 regulates the invasion and migration of oral cancer cells at a concentration that does not exert considerable toxicity to the cancer cells. Furthermore, this research on LPA and STAT3 could serve as important basis for exploring oral cancer treatment and novel biomarkers. Therefore, STAT3 plays an important role in LPA-induced oral cancer cell invasion. In a follow-up study, we are currently exploring whether the release of IL- 6 and IL- 8 and the type of G proteincoupled receptor responsible for STAT3 induces STAT3 phosphorylation in oral cancer cells. Furthermore, cancer metastasis is closely related to the survival of oral cancer patients, and the association between LPA and STAT3 could serve as important basis for exploring oral cancer treatment and novel biomarkers. Subsequent studies should investigate whether STAT3 affects LPA-induced cancer cell invasion and metastasis by conducting animal studies and using clinical data.

\section{Notes}

\section{Conflict of interest}

No potential conflict of interest relevant to this article was reported.

\section{Ethical approval}

We did not collect or record any personally identifiable 
information in this research, so this is not the case for IRB review.

\section{ORCID}

Zi Hae Song, https://orcid.org/0000-0002-6715-9126

Kyung Hwa Cho, https://orcid.org/0000-0002-3829-8194

Jin Young Kim, https://orcid.org/0000-0002-0555-4921

Hoi Young Lee, https://orcid.org/0000-0003-1954-7981

\section{Acknowledgements}

This report was received by a grant from Basic Science Research Program through the National Research Foundation of Korea (NRF) funded by the Ministry of Education, Science and Technology (NRF-2017R1E1A1 A01074091, 2017R1A2B4007361).

\section{References}

1. Xie C, Ji N, Tang Z, Li J, Chen Q: The role of extracellular vesicles from different origin in the microenvironment of head and neck cancers. Mol Cancer 18: 83, 2019. https://doi.org/10.1186/s12943-019-0985-3

2. Vokes EE, Weichselbaum RR, Lippman SM, Hong WK: Head and neck cancer. N Engl J Med 328: 184-194, 1993. https://doi.org/10.1056/NEJM199301213280306

3. Bray F, Ferlay J, Soerjomataram I, Siegel RL, Torre LA, Jemal A: Global cancer statistics 2018: GLOBOCAN estimates of incidence and mortality worldwide for 36 cancers in 185 countries. CA Cancer J Clin 68: 394-424, 2018. https://doi.org/10.3322/caac.21492

4. Dhanuthai K, Rojanawatsirivej S, Thosaporn W, et al.: Oral cancer: a multicenter study. Med Oral Patol Oral Cir Bucal 23: e23-e29, 2018. https://doi.org/10.4317/medoral.21999

5. Kumar M, Nanavati R, Modi TG, Dobariya C: Oral cancer: etiology and risk factors: a review. J Cancer Res Ther 12: 458-463, 2016. https://doi.org/10.4103/0973-1482.186696

6. Han JH, Kim EK, Lim SH, Kim CH: Literature review on the incidence and risk factor of oral cancer. J Dent Hyg Sci 12: 451-458, 2012.

7. Bell RB, Kademani D, Homer L, Dierks EJ, Potter BE: Tongue cancer: Is there a difference in survival compared with other subsites in the oral cavity? J Oral Maxillofac Surg 65: 229-236, 2007. https://doi.org/10.1016/j.joms.2005.11.094

8. Woolgar JA, Rogers SN, Lowe D, Brown JS, Vaughan ED: Cervical lymph node metastasis in oral cancer: the importance of even microscopic extracapsular spread. Oral Oncol 39: 130-137, 2003. https://doi.org/10.1016/S1368-8375(02)00030-1

9. van Leeuwen FN, Giepmans BN, van Meeteren LA, Moolenaar WH: Lysophosphatidic acid: mitogen and motility factor. Biochem Soc Trans 31(Pt 6): 1209-1212, 2003. https://doi.org/10.1042/bst0311209

10. Panupinthu N, Lee HY, Mills GB: Lysophosphatidic acid production and action: critical new players in breast cancer initiation and progression. Br J Cancer 102: 941-946, 2010. https://doi.org/10.1038/sj.bjc.6605588

11. Hwang YS, Lee SK, Park KK, Chung WY: Secretion of IL-6 and IL-8 from lysophosphatidic acid-stimulated oral squamous cell carcinoma promotes osteoclastogenesis and bone resorption. Oral Oncol 48: 40-48, 2012. https://doi.org/10.1016/j.oraloncology.2011.08.022

12. Cho KH: Lysophosphatidic acid-induced TWIST1 and slug expression in oral cancer cell invasion. J Dent Hyg Sci 17: 433-438, 2017. https://doi.org/10.17135/jdhs.2017.17.5.433

13. Kim JY, Cho KH, Lee HY: Effect of resveratrol on oral cancer cell invasion induced by lysophosphatidic acid. J Dent Hyg Sci 18: 188-193, 2018. https://doi.org/10.17135/jdhs.2018.18.3.188

14. Siveen KS, Sikka S, Surana R, et al.: Targeting the STAT3 signaling pathway in cancer: role of synthetic and natural inhibitors. Biochim Biophys Acta 1845: 136-154, 2014. https://doi.org/10.1016/j.bbcan.2013.12.005

15. Kim BH, Yi EH, Ye SK: Signal transducer and activator of transcription 3 as a therapeutic target for cancer and the tumor microenvironment. Arch Pharm Res 39: 1085-1099, 2016. https://doi.org/10.1007/s12272-016-0795-8

16. Zhao Y, Zhang J, Xia H, et al.: Stat3 is involved in the motility, metastasis and prognosis in lingual squamous cell carcinoma. Cell Biochem Funct 30: 340-346, 2012. https://doi.org/10.1002/cbf.2810

17. Wang TY, Yu CC, Hsieh PL, Liao YW, Yu CH, Chou MY: GMI ablates cancer stemness and cisplatin resistance in oral carcinomas stem cells through IL-6/Stat3 signaling inhibition. Oncotarget 8: 70422-70430, 2017. https://doi.org/10.18632/oncotarget.19711 
18. Seo JH, Jeong KJ, Oh WJ, et al.: Lysophosphatidic acid induces STAT3 phosphorylation and ovarian cancer cell motility: their inhibition by curcumin. Cancer Lett 288 : 50-56, 2010. https://doi.org/10.1016/j.canlet.2009.06.023

19. Gkouveris I, Nikitakis N, Avgoustidis D, Karanikou M, Rassidakis G, Sklavounou A: ERK1/2, JNK and STAT3 activation and correlation with tumor differentiation in oral SCC. Histol Histopathol 32: 1065-1076, 2017.

https://doi.org/10.14670/HH-11-868
20. Zhou X, Ren Y, Liu A, et al.: STAT3 inhibitor WP1066 attenuates miRNA-21 to suppress human oral squamous cell carcinoma growth in vitro and in vivo. Oncol Rep 31: 2173-2180, 2014. https://doi.org/10.3892/or.2014.3114

21. Brusevold IJ, Tveteraas IH, Aasrum M, Ødegård J, Sandnes DL, Christoffersen T: Role of LPAR3, PKC and EGFR in LPA-induced cell migration in oral squamous carcinoma cells. BMC Cancer 14: 432, 2014.

https://doi.org/10.1186/1471-2407-14-432 